ELECTRONIC RESEARCH ANNOUNCEMENTS OF THE AMERICAN MATHEMATICAL SOCIETY

Volume 8, Pages 1-10 (March 13, 2002)

S $1079-6762(02) 00099-9$

\title{
AUTOMORPHISMS OF CATEGORIES OF FREE ALGEBRAS OF VARIETIES
}

\author{
G. MASHEVITZKY, B. PLOTKIN, AND E. PLOTKIN \\ (Communicated by Efim Zelmanov)
}

\begin{abstract}
Let $\Theta$ be an arbitrary variety of algebras and let $\Theta^{0}$ be the category of all free finitely generated algebras from $\Theta$. We study automorphisms of such categories for special $\Theta$. The cases of the varieties of all groups, all semigroups, all modules over a noetherian ring, all associative and commutative algebras over a field are completely investigated. The cases of associative and Lie algebras are also considered. This topic relates to algebraic geometry in arbitrary variety of algebras $\Theta$.
\end{abstract}

\section{Motivations}

1.1. The main problem and automorphisms of free objects. We consider an arbitrary variety of algebras $\Theta$. For any $\Theta$ denote by $\Theta^{0}$ the category of all free in $\Theta$ algebras $W=W(X)$, where $X$ is finite. In order to avoid set-theoretic problems we view all $X$ as subsets of a universal infinite set $X^{0}$.

Our main goal is to study automorphisms of the category $\Theta^{0}$ and the corresponding group Aut $\Theta^{0}$.

The study of automorphisms of the category $\Theta^{0}$ is tied to the study of automorphisms of the semigroups End $W, W \in \mathrm{Ob} \Theta^{0}$. The group of automorphisms Aut $W$ consists of invertible elements of the semigroup End $W$. There is the embedding Aut $W \rightarrow \operatorname{Aut}($ End $W)$. The image of Aut $W$ is the group of all inner automorphisms of the semigroup End $W$.

A great deal is known about the group Aut $W$ for different varieties $\Theta$ and $W \in \mathrm{Ob} \Theta^{0}$. Automorphisms of free groups are well known [13], and the same is true for free Lie algebras [8], free associative algebras over a field (when the number of generators is $\leq 2$; see [8, 14, 7, 17]), and some other varieties. For free associative algebras with a greater number of generators the question is still open (see Cohn's conjecture [8]).

The relevant question is how do the towers of automorphisms of free objects look like. Let $W$ be a free object and consider the tower of groups Aut $W, \operatorname{Aut}^{2} W=$ $\operatorname{Aut}(\operatorname{Aut} W), \ldots, \operatorname{Aut}^{n} W=\operatorname{Aut}\left(\operatorname{Aut}^{(n-1)} W\right), \ldots$ Minimum $n$ such that every

Received by the editors July 4, 2001.

2000 Mathematics Subject Classification. Primary 08A35, 08CO5, 14A22, 14A99.

Key words and phrases. Algebraic variety, variety of algebras, category, free algebra, automorphisms.

This work was supported in part by the Israel Science Foundation founded by the Israel Academy of Sciences and Humanities - Center of Excellence Program and by Intas grant "Algebraic $K$-theory, groups and algebraic homotopy theory". 
automorphism of $\mathrm{Aut}^{n} W$ is inner is called the height of the tower. The heights are known for the variety of semigroups, the variety of all groups [9, 10], the category of free modules over a field or over "good" rings [1 12, etc.

There is an embedding $\tau_{W}: \operatorname{Aut}(\operatorname{End} W) \rightarrow \operatorname{Aut}(\operatorname{Aut} W)$. Investigation of $\operatorname{Ker} \tau_{W}$ and $\operatorname{Img} \tau_{W}$ is of independent interest. Formanek [10] has shown that if $\Theta$ is the variety of groups, then $\operatorname{Ker} \tau_{W}=1$, and the group $\operatorname{Aut}($ End $W$ ) is isomorphic to $\operatorname{Aut}(\operatorname{Aut} W)$. In the cases of modules or associative algebras the situation is more complicated.

Thus, there is much information related to automorphisms of individual free objects. We note that our aim is to study not these automorphisms but automorphisms of categories of free objects. It turns out that new notions have arisen which make this subject quite natural and highly motivated. We give the corresponding explanations in the next two subsections.

1.2. Geometric motivation. Our primary interest in automorphisms of categories has grown from the universal algebraic geometry (see 18, 22, 21, 20, 2, 3, 16, etc). In order to make the exposition self-contained we recall the necessary information. In this subsection we provide a glimpse on the motivation, and in the next one there will be a sketch of the subject with some precise definitions. Most of the material from 1.2 and 1.3 is collected in [18].

Let $\Theta$ be the variety of all associative, commutative algebras over the infinite ground field $P$. Denote by $W(X)=P[X], X=\left\{x_{1}, \ldots, x_{n}\right\}$, the algebra of polynomials with commuting variables, which is a free algebra in $\Theta$. The classical algebraic geometry is associated with this variety, and for any extension $L$ of the ground field $P$ the algebraic sets in the affine space $L^{n}$ correspond to the $L$-closed ideals in $P[X]$. Now suppose $L_{1}$ and $L_{2}$ are two extensions of the ground field $P$.

The key question is when do the geometries defined by $L_{1}$ and $L_{2}$ coincide? Let us denote by $K_{\Theta}(L)$ the category of all algebraic sets in $L^{n}$. This category is regarded as an invariant which is responsible for the geometry in $L$.

Then the question can be reformulated as follows: when are the categories of algebraic sets $K_{\Theta}\left(L_{1}\right)$ and $K_{\Theta}\left(L_{2}\right)$ isomorphic?

Within last years it has been figured out that one can replace the variety of associative commutative algebras (the so-called classical variety) by an arbitrary variety of algebras $\Theta$ and construct algebraic geometry in $\Theta$ with respect to a distinguished algebra $H$ in $\Theta$. This $H$ takes the role of the field $L$. Thus, let $\Theta$ be an arbitrary variety of algebras, $H_{1}, H_{2}$ algebras in $\Theta$, and $K_{\Theta}\left(H_{1}\right), K_{\Theta}\left(H_{2}\right)$ the corresponding categories of algebraic sets.

The principal problem for the variety $\Theta$ repeats the one for the classical case:

Problem 1.1. When do the geometries over $H_{1}$ and $H_{2}$ coincide, i.e., when are the categories $K_{\Theta}\left(H_{1}\right)$ and $K_{\Theta}\left(H_{2}\right)$ isomorphic?

There is an answer to this question [18, which is formulated in terms of two notions: geometric equivalence and geometric similarity of algebras (see 1.3).

Geometric similarity provides necessary and sufficient conditions for the categories $K_{\Theta}\left(H_{1}\right)$ and $K_{\Theta}\left(H_{2}\right)$ to be isomorphic, while geometric equivalence gives only a sufficient condition. However, the notion of geometric equivalence is much more explicit, transparent and well verified than the notion of geometric similarity. Thus, the main problem is converted to the following: 
Problem 1.2. For which categories $\Theta$ does the geometric similarity either coincide with geometric equivalence or is close to it?

We show in 1.3 that this problem is tied to the description of automorphisms of the category of free algebras in $\Theta$.

1.3. Basics of universal algebraic geometry. Fix an algebra $H$ in $\Theta$. Any equation in $W(X),|X|=n$ has the form $w=w^{\prime}, w, w^{\prime} \in W$. Systems of equations in $W$ are denoted by $T$. They can be viewed as binary relations in $W$. The set of homomorphisms $\operatorname{Hom}(W, H)$ is regarded as an affine space. There is the canonical bijection $\operatorname{Hom}(W, H) \simeq H^{n}$. A point $\mu: W \rightarrow H$ is a solution of equation $w=w^{\prime}$ if and only if $\left(w, w^{\prime}\right) \in \operatorname{Ker} \mu$. Consider sets of points $A \subset \operatorname{Hom}(W, H)$. The Galois correspondence between systems of equations $T$ and sets $A$ is given by:

$$
\left\{\begin{array}{l}
T^{\prime}=A=\{\mu: W \rightarrow H \mid T \subset \operatorname{Ker} \mu\}=T_{H}^{\prime}, \\
A^{\prime}=T=\bigcap_{\mu \in A} \operatorname{Ker} \mu .
\end{array}\right.
$$

Definition 1.3. Algebras $H_{1}$ and $H_{2}$ are called geometrically equivalent if for every finite set $X$ and every system of equations $T$ in $W=W(X)$ the equality

$$
T_{H_{1}}^{\prime \prime}=T_{H_{2}}^{\prime \prime}
$$

holds.

Definition 1.4. 1. A set $A$ such that $A=T^{\prime}$ for some $T$ is called an algebraic set.

2. A congruence $T$ in $W$ is called $H$-closed if there exists an algebraic set $A$ such that $T=A^{\prime}$.

Denote by $\mathrm{Cl}_{H}(W)$ the set of all $H$-closed congruences in $W$. This gives rise to the contravariant functor $\mathrm{Cl}_{H}: \Theta^{0} \rightarrow$ Set. Thus, we can reformulate Definition 1.3 in terms of the functors $\mathrm{Cl}_{H}$, i.e., the geometric equivalence of algebras $H_{1}$ and $H_{2}$ means that the functors $\mathrm{Cl}_{H_{1}}$ and $\mathrm{Cl}_{H_{2}}$ coincide.

The geometric equivalence is a quite nice property, which in many cases can be checked effectively:

Theorem 1.5 ([21]). If algebras $H_{1}$ and $H_{2}$ are geometrically equivalent, then they have the same quasiidentities.

In the classical case the if and only if statement is true. However, for arbitrary $\Theta$ the converse statement is not valid; see [16, 11. For special categories the situation is even more transparent:

Theorem 1.6 (4]). Two abelian groups $H_{1}$ and $H_{2}$ are geometrically equivalent if and only if

1. They have the same exponents.

2. For every prime $p$ the exponents of Sylow subgroups $H_{1 p}$ and $H_{2 p}$ coincide.

An easy, but crucial fact states that the geometric equivalence of algebras $H_{1}$ and $H_{2}$ gives a sufficient condition for the categories of algebraic sets $K_{\Theta}\left(H_{1}\right)$ and $K_{\Theta}\left(H_{2}\right)$ to be isomorphic.

In order to get a necessary and sufficient condition we have to use the notion of geometric similarity. Let $\operatorname{Var}\left(H_{1}\right)$ and $\operatorname{Var}\left(H_{2}\right)$ be the varieties generated by $H_{1}$ and $H_{2}$, respectively; for simplicity we assume that $\operatorname{Var}\left(H_{1}\right)=\operatorname{Var}\left(H_{2}\right)=\Theta$. 
Geometric similarity of algebras means that there is an isomorphism

$$
\varphi: \operatorname{Var}\left(H_{1}\right)^{0} \rightarrow \operatorname{Var}\left(H_{2}\right)^{0}
$$

with the commutative diagram

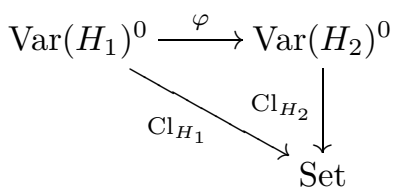

Commutativity of the diagram indicates that there is the isomorphism (not necessarily equality) of the functors $\mathrm{Cl}_{H_{1}}$ and $\mathrm{Cl}_{H_{2}} \varphi$. This isomorphism $\alpha=\alpha(\varphi)$ depends on the isomorphism of categories $\varphi$ and is constructed in a special way.

The notion of geometric equivalence is a particular case of geometric similarity when $\varphi=1$. The principal observation [18] says that if the isomorphism $\varphi$ is isomorphic as a functor to the identity functor, then geometric similarity implies geometric equivalence. Thus, we have come to the fact which lies in the basis of investigation of automorphisms of categories of free algebras: if in the category $\Theta^{0}$ every automorphism is isomorphic to the identity functor and $\operatorname{Var}\left(H_{1}\right)=\operatorname{Var}\left(H_{2}\right)=\Theta$, then the geometries over algebras $H_{1}$ and $H_{2}$ coincide if and only if the algebras $H_{1}$ and $H_{2}$ are geometrically equivalent.

\section{Definitions}

2.1. Hereditary automorphisms of categories. Let $C$ be an arbitrary (possibly small) category. Let End $C$ be the semigroup of all covariant endofunctors of the category $C$. We use the word "endomorphisms" instead of "endofunctors". A functor $\varphi: C \rightarrow C$ is called an automorphism of the category $C$ if there exists a functor $\varphi^{-1}: C \rightarrow C$ such that $\varphi \varphi^{-1}=\varphi^{-1} \varphi=1_{C}$, where $1_{C}$ is the identity functor of $C$. All automorphisms of the category $C$ form a group denoted by Aut $C$.

Two functors are called isomorphic if there exists an invertible natural transformation of functors which takes one to the other. Thus, the relation of isomorphism of functors is defined on the semigroup End $C$. This relation turns out to be a congruence of End $C$. The quotient semigroup is denoted by $\operatorname{End}^{0}(C)$. The group of invertible elements of $\operatorname{End}^{0}(C)$ is denoted by $\operatorname{Aut}^{0}(C)$. The group $\operatorname{Aut}^{0}(C)$ is the group of all autoequivalences of the category $C$ which are considered up to an isomorphism of functors. There is the canonical homomorphism $\tau:$ Aut $C \rightarrow \operatorname{Aut}^{0} C$. The kernel of $\tau$ consists of automorphisms isomorphic to the identity functor (inner automorphisms; see 2.2). It is not clear for what categories the homomorphism $\tau$ is surjective.

Definition 2.1. An automorphism $\varphi: C \rightarrow C$ is called hereditary if for every $A \in \mathrm{Ob} C$ the objects $A$ and $\varphi(A)$ are isomorphic.

It is clear that an automorphism $\varphi: C \rightarrow C$ induces an isomorphism of the semigroups End $A$ and End $\varphi(A)$, and of the groups Aut $A$ and Aut $\varphi(A)$. This implies immediately that every automorphism of the categories of finite sets or free semigroups is hereditary.

A finitely generated free in $\Theta$ algebra $W=W(X)$ is hopfian if every surjection $W \rightarrow W$ turns out to be an automorphism of $W$. 
Definition 2.2. A variety $\Theta$ is called hopfian if every finitely generated free algebra in $\Theta$ is hopfian.

Denote by $W_{0}=W\left(x_{0}\right)$ the free cyclic algebra with the generator $x_{0}$.

Proposition 2.3. If $\Theta$ is a hopfian variety and the algebras $W_{0}$ and $\varphi\left(W_{0}\right)$ are isomorphic, then $\varphi$ is a hereditary automorphism of the category $\Theta^{0}$.

Definition 2.4. The category $C$ is called automorphic hereditary if each of its automorphisms is hereditary.

Remark 2.5. The categories of sets, free semigroups, free groups, free modules over a noetherian ring, free associative commutative algebras are automorphic hereditary. However, not every category is automorphic hereditary.

2.2. Inner automorphisms. Let $\varphi$ be a substitution on objects of the category $C$ such that $A$ and $\varphi(A)$ are isomorphic for every $A \in \mathrm{Ob} C$. Consider a function $s$ which for any object $A$ chooses an isomorphism

$$
s_{A}: A \rightarrow \varphi(A) .
$$

Define an automorphism $\hat{s}: C \rightarrow C$ by the rule:

1. $\hat{s}(A)=\varphi(A)$, for every object $A$.

2. For every morphism $\nu: A \rightarrow B$,

$$
\hat{s}(\nu)=s_{B} \nu s_{A}^{-1}: \varphi(A) \rightarrow \varphi(B) .
$$

Definition 2.6. An automorphism $\varphi: C \rightarrow C$ is called inner if

1. $\varphi$ is a hereditary automorphism.

2. For the substitution $\varphi$ there exists a function $s$ such that $\varphi=\hat{s}$.

The equality $\hat{s}(\nu)=\varphi(\nu)=s_{B} \nu s_{A}^{-1}$ can be written as a commutative diagram,

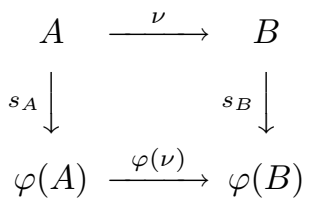

This diagram means that the natural transformation of functors $s: 1_{c} \rightarrow \varphi$ is an isomorphism of functors. Thus, an automorphism $\varphi: C \rightarrow C$ is inner if and only if $\varphi$ is isomorphic to the identity automorphism $1_{c}: C \rightarrow C$. Note that two automorphisms $\varphi_{1}, \varphi_{2}: C \rightarrow C$ are isomorphic if and only if $\varphi_{1}^{-1} \varphi_{2}$ is inner.

Proposition 2.7. All inner automorphisms form a normal subgroup in Aut $C$ denoted by $\operatorname{Int} C$.

Now one can define the group Out $C$ of outer automorphisms of the category $C$ by Out $C=\operatorname{Aut} C / \operatorname{Int} C$.

Definition 2.8. The category $C$ is called perfect if every automorphism of it is inner.

Thus, a category $C$ is perfect if and only if Out $C=1$. Thus, if we consider automorphisms $C$ up to isomorphisms, a perfect $C$ has no automorphisms except trivial.

Proposition 2.9. Every hereditary automorphism $\varphi$ of the category $C$ can be presented in the form $\varphi=\varphi_{1} \varphi_{2}$, where $\varphi_{1}$ is an inner automorphism and $\varphi_{2}$ is an automorphism which does not change objects. 
Let us call an automorphism $\varphi$ which does not change objects a stable automorphism.

Denote by HAut $C$ the normal subgroup of all hereditary automorphisms and by St $C$ the normal subgroup of all automorphisms which does not change objects of $C$. Then HAut $C=\operatorname{Int} C \cdot \operatorname{St} C$. For the automorphic hereditary categories, HAut $C=$ Aut $C$ and Aut $C=\operatorname{Int} C \cdot \operatorname{St} C$, respectively.

2.3. Remarks. First of all observe that if an automorphism $\varphi$ of the category $C$ is stable, then it induces the automorphism $\varphi_{A}$ of the semigroup End $A$ and of the groups Aut $A$ for any object $A \in C$. Thus, we get homomorphisms $\operatorname{St} C \rightarrow$ $\operatorname{Aut}($ End $A)$ and $\operatorname{St} C \rightarrow \operatorname{Aut}(\operatorname{Aut} A)$ and a description of lower floors of towers of automorphisms of free objects becomes of special importance.

An object $A \in \mathrm{Ob} C$ is called perfect if every automorphism of the semigroup End $A$ is inner. If $\varphi \in \operatorname{St} C$ and $\varphi$ is inner, then $\varphi_{A}$ is an inner automorphism of End $A$. On the other hand, if $\psi$ is an inner automorphism of End $A$, then $\psi=\varphi_{A}$ for some $\varphi \in \operatorname{St} C$. Hence, if $A$ is a perfect object of $C$, then the homomorphism St $C \rightarrow \operatorname{Aut}($ End $A$ ) is surjective.

Note that perfectness of $C$ does not imply that every object of $C$ is perfect. On the other hand, perfectness of each object is an argument in favor of the perfectness of the category.

\section{The MAIN THEOREM}

3.1. Category $\Theta^{0}$. Recall that for any variety of algebras $\Theta$, the category $\Theta^{0}$ is the category of all free finitely generated algebras in $\Theta$.

Definition 3.1. A variety $\Theta$ is called automorphic hereditary if the category $\Theta^{0}$ is automorphic hereditary, i.e., if every automorphism $\varphi: \Theta^{0} \rightarrow \Theta^{0}$ is hereditary.

A variety $\Theta$ is called regular if for every $X, Y$ an isomorphism $W(X) \simeq W(Y)$, where algebras $W(X), W(Y)$ are free in $\Theta$, implies $|X|=|Y|$.

A variety $\Theta$ is called noetherian if every finitely generated free algebra $W=$ $W(X)$ is noetherian with respect to congruences.

It is clear that every noetherian variety is hopfian, and hence regular.

Definition 3.2. A variety $\Theta$ is called perfect if the category of free algebras $\Theta^{0}$ is perfect, i.e., if every automorphism $\varphi: \Theta^{0} \rightarrow \Theta^{0}$ is inner.

A variety $\Theta$ is called almost perfect if the group Out $\Theta^{0}$ is finite.

3.2. Algebras with constants. The main geometrical applications require the existence of constants in the algebras under consideration. In this section we introduce the corresponding notions.

Let $\Theta$ be an arbitrary variety of algebras, and $G$ a distinguished nontrivial algebra in $\Theta$. Consider the category $\Theta^{G}$ whose objects have the form $h: G \rightarrow H$, where $H \in \Theta$ and $h$ is a morphism in $\Theta$. Morphisms in $\Theta^{G}$ are presented by commutative diagrams

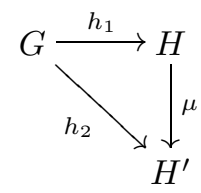


where $\mu, h_{1}, h_{2}$ are morphisms in $\Theta$. Objects of $\Theta^{G}$ are called $G$-algebras and are denoted by $(H, h)$. Elements of $G$ have the meaning of constants in algebras from $\Theta$ and, adding them as nullary operations to the signature of $\Theta$, we get the variety of $G$-algebras $\Theta^{G}$.

A free in $\Theta^{G}$ algebra $W=W(X)$ has the form of the free product $G * W_{0}(X)$, where $W_{0}(X)$ is a free algebra in $\Theta$.

Examples. 1. The variety of commutative associative algebras over a field $P$ is of type $\Theta^{G}$, where $\Theta$ is the variety of associative commutative rings with 1 , and $G$ is the field $P$.

2. The variety of associative algebras over a field.

3. The variety of $G$-groups.

The category $\Theta^{G}$ is a subcategory in the category $\Theta(G)$ with the same objects, while the morphisms of $\Theta(G)$ are presented by the commutative squares

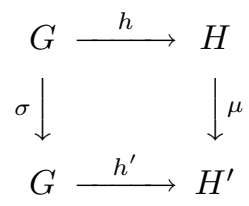

where $\sigma \in \operatorname{End} G$.

Morphisms of the category $\Theta(G)$ are called semimorphisms of the initial category of algebras with constants $\Theta^{G}$.

Consider the category $\left(\Theta^{G}\right)^{0}$ of free $G$-algebras.

Definition 3.3. An automorphism of $\left(\Theta^{G}\right)^{0}$ is called semiinner if it is induced by an inner automorphism of the category $\Theta(G)^{0}$.

This means that a semiinner automorphism $\varphi$ of the category $\left(\Theta^{G}\right)^{0}$ is given by a pair $(\sigma, s)$, where $\sigma$ is an automorphism of the algebra $G$, and $s$ is a function which attaches to a finite set $X$ a semiisomorphism $\left(\sigma, s_{X}\right): W(X) \rightarrow \varphi W(X)$. The automorphism $\sigma$ does not depend on $X$.

All semiinner automorphisms of the category $\left(\Theta^{G}\right)^{0}$ constitute a subgroup in $\operatorname{Aut}\left(\Theta^{G}\right)^{0}$ denoted by $\operatorname{SInt}\left(\Theta^{G}\right)^{0}$. If this subgroup has a finite index in $\operatorname{Aut}\left(\Theta^{G}\right)^{0}$ then the category $\left(\Theta^{G}\right)^{0}$ is called almost semiperfect. The variety $\left(\Theta^{G}\right)$ is almost semiperfect if the category $\left(\Theta^{G}\right)^{0}$ is almost semiperfect.

Remark. The definitions above do not cover the case of the category of free modules over a ring $R$ since there is no canonical embedding of $R$ to a module. However, the standard definition of semiautomorphisms of a free module has the same meaning.

Let $\sigma$ be an automorphism of a ring, and $K X=K x_{1} \oplus \cdots \oplus K x_{n}$ a free module. Define $\sigma_{X}: K X \rightarrow K X$ by the rule $\sigma_{X}(u)=\lambda_{1}^{\sigma} x_{1}+\cdots+\lambda_{n}^{\sigma} x_{n}$, where $u=$ $\lambda_{1} x_{1}+\cdots+\lambda_{n} x_{n}$ is an element of $K X$. A pair $\left(\sigma, \sigma_{X}\right)$ is called a semiautomorphism of $K X$.

Now, we can consider the category of modules with semimorphisms (semilinear maps). In this category there are inner morphisms. The morphisms of the category of modules induced by inner morphisms of the category of modules with semimorphisms are called semiinner morphisms of the category of modules.

Definition 3.4. A variety $\Theta^{G}$ is called semiperfect if every automorphism of the category $\left(\Theta^{G}\right)^{0}$ is semiinner. 
Definition 3.5. $G$-algebras $\left(H_{1}, h_{1}\right)$ and $\left(H_{2}, h_{2}\right)$ are called geometrically semiequivalent if there exists an algebra $(H, h)$ such that $\left(H_{1}, h_{1}\right)$ and $(H, h)$ are semiisomorphic and $(H, h)$ is geometrically equivalent to $\left(H_{2}, h_{2}\right)$.

Theorem $3.6([18])$. If the geometric similarity of $G$-algebras $\left(H_{1}, h_{1}\right)$ and $\left(H_{2}, h_{2}\right)$ is given by a semiinner automorphism, then they are geometrically semiequivalent.

\subsection{The main theorem.}

Theorem 3.7. 1. The categories of sets and finite sets are perfect.

2. The variety of all groups is perfect.

3. The variety of all semigroups is almost perfect.

4. The variety of all $R$-modules, where $R$ is a noetherian ring, is semiperfect.

5. The variety of commutative associative algebras with unity element over an infinite field, is semiperfect [4].

6. The variety of F-groups, where $F$ is a free group, is semiperfect.

Corollary 3.8. 1. Let $H_{1}, H_{2}$ be two groups, and let each of them generate the variety of all groups. The categories of algebraic sets $K_{\Theta}\left(H_{1}\right)$ and $K_{\Theta}\left(H_{2}\right)$ are isomorphic if and only if the groups are geometrically equivalent.

2. An F-group $(H, h)$ is called faithful if $h$ is a monomorphism. Let $H_{1}, H_{2}$ be two faithful $F$-groups. Then the corresponding categories of algebraic sets are isomorphic if and only if the F-groups are geometrically semiequivalent.

3. The same is true for modules over a noetherian ring $R$ and for commutative associative algebras over an infinite field.

Problem 3.9. Describe automorphisms of the categories of free associative and free Lie algebras.

3.4. Sketch of the proof. 1. The result for the categories of sets and finite sets is relatively easy and is based on the ideas from [24].

2. We prove that all varieties from the Main Theorem are hereditary automorphic. This implies that we can study only stable automorphisms. It can be proven that every such automorphism $\varphi$ is a quasiinner automorphism. This means that there is a function $\sigma=\sigma(\varphi)$ which for every finite $X$ takes a bijection $\sigma_{X}: W(X) \rightarrow W(X)$, and such that $\varphi(\nu)=\sigma_{Y} \nu \sigma_{X}^{-1}$ for every $\nu: W(X) \rightarrow W(Y)$.

3. Let $\Theta$ be the variety of all groups. By Formanek's theorem [10], every automorphism of the semigroup End $W(X),|X|>1$, is an inner automorphism. Using this result it can be proven that the function $\sigma$ is presented in the form $\sigma=s \tau$, where $s_{X}$ is an automorphism of the group $W(X)$, and $\tau$ is either the identity function or $\tau_{X}(a)=a^{-1}$ for every finite $X$ and every $a \in W(X)$. Since $\tau$ is a central function, it disappears and therefore $\hat{\sigma}=\hat{s}$. For every $\nu: W(X) \rightarrow W(Y)$, we have $\varphi(\nu)=s_{Y} \nu s_{X}^{-1}$. Hence, $\varphi$ is an inner automorphism.

4. The case of semigroups. Let $F=F(X)$ be a free semigroup and $u=$ $x_{i_{1}} x_{i_{2}} \cdots x_{i_{n-1}} x_{i_{n}}$ an element of $F$. Denote by $\bar{u}$ the element $\bar{u}=x_{i_{n}} x_{i_{n-1}} \cdots x_{i_{2}} x_{i_{1}}$. The map $u \rightarrow \bar{u}$ is a bijective involution on the set $F(X)$.

Now we can define an automorphism $\mu$ of the category $\Theta^{0}$ of free semigroups. This automorphism does not change objects, and for every $\nu: F(X) \rightarrow F(Y)$ we set $\mu(\nu)(x)=\overline{\nu(x)}$ for every $x \in X$. Automorphism $\mu$ is called a mirror automorphism of the category $\Theta^{0}$. It is clear that $\mu^{2}=i d$. The mirror automorphism of the semigroups End $F(X)$ is defined similarly. 
Using [15] it can be proved that any automorphism $\varphi$ of the category $\Theta^{0}$ can be presented as the product of inner and mirror automorphisms. Obviously, Out $\Theta^{0}$ is isomorphic to $Z_{2}$.

5. Let $\Theta$ be an arbitrary hopfian variety of algebras, and let it be generated by a cyclic free algebra $W=W_{0}=W\left(x_{0}\right)$. Consider an automorphism $\varphi$ of the category $\Theta^{0}$ which does not change objects. Denote by $\varphi_{W_{0}}$ the automorphism of the semigroup End $W\left(x_{0}\right)$ induced by the automorphism $\varphi$. The following theorem holds:

Theorem 3.10. If the automorphism $\varphi_{W_{0}}$ is trivial, then $\varphi$ is an inner automorphism of the category $\Theta^{0}$.

6. Let us use the theorem above in the case of modules. Let $\Theta$ be the variety of modules over a noetherian ring $R$ and $\varphi$ an automorphism which does not change objects. Take the cyclic module $R x_{0}$. It generates the whole variety $\Theta$. It can be proven that $\varphi$ induces an automorphism of the ring $R$. The corresponding $\varphi_{R x_{0}}$ is a semiinner automorphism of End $R x_{0}$, which can be extended to a semiinner automorphism $\psi$ of the category $\Theta^{0}$. The automorphism $\psi^{-1} \varphi$ acts trivially in the semigroup $\operatorname{End}\left(R x_{0}\right)$. Therefore $\psi^{-1} \varphi$ is inner. Hence, $\varphi$ is semiinner.

7. The case of associative commutative algebras follows the scheme of item 6 . The same scheme works for the situation of $F$-groups.

8. About Problem 3.9. Consider a generalization of Theorem 3.10.

Let $\Theta$ be an arbitrary hopfian variety of algebras, and let $\Theta$ be generated by an algebra $W^{0}=W\left(X^{0}\right)$, where $X^{0}$ is a fixed finite set. Denote by $W_{0}=W\left(x_{0}\right)$ the cyclic free algebra. Let $\nu_{0}: W^{0} \rightarrow W_{0}$ be a morphism defined by the condition: $\nu_{0}(x)=x_{0}$ for every $x \in X^{0}$.

Theorem 3.11. If the automorphism $\varphi: \Theta^{0} \rightarrow \Theta^{0}$ acts trivially on the semigroups End $W^{0}$ and End $W_{0}$ and $\varphi\left(\nu_{0}\right)=\nu_{0}$, then $\varphi$ is an inner automorphism of the category $\Theta^{0}$.

9. Let $\Theta$ be the variety of associative or Lie algebras over a field, $F_{0}$ the free algebra with one variable, $F^{0}$ the free algebra with two variables. Consider a full subcategory of $\Theta^{0}$ which has only two objects $F_{0}$ and $F^{0}$ and with morphisms induced by the morphisms of $\Theta^{0}$.

The theorem above allows us to reduce the problem on automorphisms of the category $\Theta^{0}$ to studying the automorphisms of this subcategory.

We note that $\Theta$ is generated by the free algebra with two variables $F^{0}$.

The notion of a mirror automorphism works in the variety of all associative algebras $\Theta$ as well.

\section{REFERENCES}

[1] Automorphisms of classical groups, Moscow, Mir, 1976.

[2] G. Baumslag, A. Myasnikov, V. Remeslennikov, Algebraic geometry over groups, J. Algebra 219 (1999), 16-79. MR 2000j:14003

[3] G. Baumslag, A. Myasnikov, V. Remeslennikov, Algebraic geometry over groups, in "Algorithmic problems in groups and semigroups", Birkhäuser, Boston, 1999, pp. 35-51. MR 2000m:20066

[4] A. Berzins, Geometric equivalence of algebras, International J. of Algebra and Computations 11:4 (2001), 447-456.

[5] A. Berzins, Variety Grp-F is semiperfect, Preprint, Riga, 1998.

[6] A. Berzins, B. Plotkin, E. Plotkin, Algebraic geometry in varieties of algebras with the given algebra of constants, Journal of Math. Sciences 102:3 (2000), 4039-4070. MR 2001h:14001 
[7] A. Czerniakiewicz, Automorphisms of a free associative algebra of rank 2. II, Trans. Amer. Math. Soc. 171 (1972), 309-315. MR 43:6269

[8] P. M. Cohn, Free rings and their relations, Academic Press, 1985. MR 87e:16006

[9] J. Dyer, E. Formanek, The automorphism group of a free group is complete, J. London Math. Soc. (2) 11:2 (1975), 181-190. MR 52:588

[10] E. Formanek, A question of B. Plotkin about the semigroup of endomorphisms of a free group, Proc. Amer. Math. Soc. 130 (2002), 935-937.

[11] R. Gobel, S. Shelah. Radicals and Plotkin's problem concerning geometrically equivalent groups, Proc. Amer. Math. Soc. 130 (2002), 673-674.

[12] L. K. Hua and I. Reiner, Automorphisms of the unimodular group, Trans. Amer. Math. Soc. 71 (1951), 331-348. MR 13:328f

[13] R. C. Lyndon, P. E. Schupp, Combinatorial group theory, Springer Verlag, 1977. MR 58:28182 MR 2001i:20064

[14] L. Makar-Limanov, The automorphisms of the free algebra with two generators, Funktsional. Anal. i Pril. 4:3 (1970), 107-108. (Russian) MR 42:6044

[15] G. Mashevitzky, A question of B. Plotkin about automorphisms of the semigroup of endomorphisms of a free semigroup, Preprint, Ben Gurion Univ., 2001.

[16] A. Myasnikov, V. Remeslennikov, Algebraic geometry over groups II. Logical foundations, J. of Algebra 234 (2000), 225-276. MR 2001i:14001

[17] M. Nagata, On the automorphism group of $k[x, y]$, Lectures in Math., Kyoto Univ., Kinokuniya, Tokyo, 1972 MR 49:2731

[18] B. Plotkin, Seven lectures in universal algebraic geometry, Preprint, Hebrew University, Jerusalem, 2000.

[19] B. Plotkin, Algebraic logic, varieties of algebras and algebraic varieties, in Proc. Int. Alg. Conf., St. Petersburg, 1995, St. Petersburg, 1999, pp. 189-271.

[20] B. Plotkin, Varieties of algebras and algebraic varieties, Israel. Math. Journal 96:2 (1996), 511-522. MR 98c:08011

[21] B. Plotkin, Varieties of algebras and algebraic varieties. Categories of algebraic varieties, Siberian Advances in Mathematics, Allerton Press, 7:2 (1997), 64-97. MR 99a:08004

[22] B. Plotkin, Some notions of algebraic geometry in universal algebra, Algebra i Analiz 9:4 (1997), 224-248; English transl., St. Petersburg Math. J. 9:4 (1998), 859-879. MR 98j:08003

[23] B. Schein and B. Teclezghi, Endomorphisms of finite full transformation semigroups, Proc. Amer. Math. Soc. 126 (1998), 2579-2587. MR 99b:20093

[24] J. Schreier, Uber Abbildungen einer abstrakten Menge auf ihre Teilmengen, Fundamenta Mathematica 28 (1936), 261-264.

Department of Mathematics, Ben Gurion University of the Negev, 84105, Israel

E-mail address: gmash@cs.bgu.ac.il

Institute of Mathematics, The Hebrew University, Jerusalem, 91904, Israel

E-mail address: borisov@math.huji.ac.il

Department of Mathematics, Bar Ilan University, Ramat Gan, 52900, Israel

E-mail address: plotkin@macs.biu.ac.il 